\title{
Papers
}

\section{Overdiagnosis of malaria in patients with severe febrile illness in Tanzania: a prospective study}

Hugh Reyburn, Redempta Mbatia, Chris Drakeley, Ilona Carneiro, Emmanuel Mwakasungula, Ombeni Mwerinde, Kapalala Saganda, John Shao, Andrew Kitua, Raimos Olomi, Brian M Greenwood, Christopher J M Whitty

\begin{abstract}
Objective To study the diagnosis and outcomes in people admitted to hospital with a diagnosis of severe malaria in areas with differing intensities of malaria transmission.

Design Prospective observational study of children and adults over the course a year.

Setting 10 hospitals in north east Tanzania.

Participants 17313 patients were admitted to hospital; of these 4474 (2851 children aged under 5 years) fulfilled criteria for severe disease.

Main outcome measure Details of the treatment given and outcome. Altitudes of residence (a proxy for transmission intensity) measured with a global positioning system.

Results Blood film microscopy showed that 2062 (46.1\%) of people treated for malaria had Plasmodium falciparum (slide positive). The proportion of slide positive cases fell with increasing age and increasing altitude of residence. Among 1086 patients aged $\geq 5$ years who lived above 600 metres, only $338(31.1 \%)$ were slide positive, while in children $<5$ years living in areas of intense transmission $(<600$ metres $)$ most (958/1392, 68.8\%) were slide positive. Among 2375 people who were slide negative, $1571(66.1 \%)$ were not treated with antibiotics and of those, $120(7.6 \%)$ died. The case fatality in slide negative patients was higher $(292 / 2412,12.1 \%)$ than for slide positive patients $(142 / 2062,6.9 \%)(\mathrm{P}<0.001)$. Respiratory distress and altered consciousness were the strongest predictors of mortality in slide positive and slide negative patients and in adults as well as children.

Conclusions In Tanzania, malaria is commonly overdiagnosed in people presenting with severe febrile illness, especially in those living in areas with low to moderate transmission and in adults. This is associated with a failure to treat alternative causes of severe infection. Diagnosis needs to be improved and syndromic treatment considered. Routine hospital data may overestimate mortality from malaria by over twofold.
\end{abstract}

\section{Introduction}

In the year 2000 about $42 \%$ of hospital diagnoses and 32\% of hospital deaths in Tanzania were attributed to malaria, ${ }^{1}$ a figure typical for countries in Africa where malaria is endemic. ${ }^{2}$ Despite this striking statistic, little is known about the accuracy of hospital diagnosis or the appropriateness of treatment. A recent study from Tanzania found that of 75 adults diagnosed and treated for cerebral malaria in a teaching hospital only two met World Health Organization criteria for the diagnosis, ${ }^{3}$ and two studies of district hospitals in Africa identified several problems with the organisation and planning of care. ${ }^{45}$

Given the high proportion of admissions attributed to malaria, overdiagnosis of malaria and consequent neglect of alternative diagnoses could lead to avoidable morbidity and mortality. In addition, overdiagnosis burdens health services with costs they can ill afford. ${ }^{6}$ Unreliable hospital data hamper health service planning and make progress towards targets such as those set by the Roll Back Malaria initiative impossible to assess. The spread of drug resistance means that there is a need to move to considerably more expensive drugs, but if a large proportion of the people treated for malaria do not have the disease this will substantially increase the costs of change.

Accuracy of hospital diagnosis of malaria is likely to depend on the epidemiological probability of the disease (defined by intensity of malaria transmission and age of patients) and is important as most of the population of sub-Saharan Africa live in areas of low or moderate malaria transmission. ${ }^{7}$ We prospectively examined the diagnosis and outcome in all patients admitted and treated for severe or potentially complicated malaria during one year in 10 hospitals serving people for areas with various transmission intensities. A clinician's decision to admit a patient for treatment of malaria defined those eligible for inclusion in the study.

\section{Methods}

The study was conducted in north east Tanzania, an area characterised by the Eastern Arc mountains with a populated altitude ranging from sea level to about 1800 metres. In this area, altitude has been shown to be a valid proxy for the intensity of malaria transmission ${ }^{8}$ with measured entomological inoculation rates of 300 infectious bites per year on the coastal plain, 30 at an altitude of 930 metres, and $<1$ above 1500 metres.

We selected hospitals that provided a well organised service and had trained staff willing to participate. This represents most government hospitals in the area. Six were highland district hospitals at altitudes ranging from 940-1450 metres, one regional and one referral hospital served a semiurban area of 200000 people at an altitude of 900-970 metres, and two were district hospitals on the coastal plain at 320 metres and 198 metres.

The study ran at nine hospital sites from 16 February 2002 to 15 February 2003, with an additional hospital for six months (from 15 August 2002). Because of the large number of admissions to the district hospital at the lowest altitude, patients aged $<13$ years were recruited on alternate days (that is, a $50 \%$ sample of paediatric admissions). At the three busiest hospitals 
research staff were based in the paediatric ward and visited other wards two to three times daily. In the seven remaining district hospitals, one dedicated clinician and a team of clinical and laboratory staff from within each hospital collected data in the course of their normal work, supported by twice weekly visits from a project clinician.

All patients admitted for the treatment of malaria were eligible for the study. After the patients (or parents) gave informed consent, researchers collected details of age, sex, and village of residence for every case. Patients were assessed for potentially severe disease based on WHO criteria for severe malaria ${ }^{9}$ with the addition of moderate anaemia. Criteria included haemoglobin $<80 \mathrm{~g} / \mathrm{l}$ (HaemoCue AB Angelhom), inability to drink or suck (observed), inability to sit unsupported in patients aged $>1$ year, inability to localise pain in patients aged $>1$ year, confusion in patients aged $>5$ year, lower chest indrawing or deep breathing, and jaundice. If any of these were present, the researcher also recorded axillary temperature, respiratory pattern and rate, and Blantyre coma score. ${ }^{10}$ Blood glucose was measured (AccuCheck Active, Roche Diagnostics) if the Blantyre coma score was $<5$. As staining methods varied between hospitals, a separate blood film was taken ("research slide") in addition to the hospital slide, though only the hospital slide result was available to contribute to the patient's care. Outcome and treatment received were recorded at discharge or death.

Research laboratory staff stained blood slides with Giemsa stain and counted the number of Plasmodium falciparum asexual parasites per 200 leucocytes. A slide was considered negative if no parasites were found after 100 high power fields were scanned. All slides were read twice independently by microscopists blind to other data. Discordant slides were read a third time. The majority result was accepted for positive/negative discrepancies, and the geometric mean density of positive readings used for parasite density. Analyses relate to the research slide result unless specified otherwise.

Villages from which people with malaria had been admitted were visited by a trained field assistant and a global positioning point for altitude, latitude, and longitude was taken from the central point in the village.

\section{Analysis}

We double entered data in Microsoft Access and used Stata 8 (StataCorp, College Station, TX) for the statistical analysis. Initial tabulations and univariate analyses examined the distribution of slide positivity and case fatality overall and within categories. We used random effects logistic regression to assess the adjusted effect of covariates on slide positivity and mortality and to adjust for correlation within hospitals. Data are presented on actual cases recruited except where logistic regression has been used and the data were weighted to adjust for the sampling of children on alternate days in one district hospital and stratified by two six month periods to allow for the hospital that was included in the study only for the latter six months.

\section{Results}

A total of 17313 cases were recruited into the study over one year (fig 1). Of these, 12643 patients had a diagnosis of malaria but did not have any study criteria for severe disease, of whom $120(1.0 \%)$ died.

In total 4670 patients had at least one of the study criteria for severe disease and were admitted to hospital and treated for malaria, in $95 \%$ of cases with quinine. Of these patients, 196 $(4.2 \%)$ had a missing or unreadable blood slide. Among the 4474 remaining patients, $2062(46.1 \%)$ had a positive blood slide as

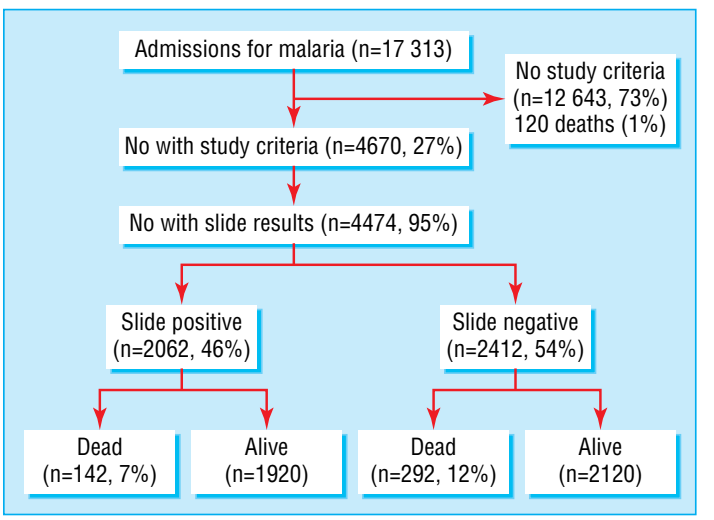

Fig 1 Patients admitted to 10 hospitals with diagnosis of malaria over one year by outcome, presence of any $P$ falciparum asexual parasites on the research blood slide, and case fatality

determined by the presence of $P$ falciparum asexual parasites on the research slide (slide positive). Most adults at every altitude band and most children under 5 years living above 600 metres had a negative slide (table 1). The proportion of patients with positive slides decreased systematically with increasing age and with increasing altitude of residence (fig 2).

When we used logistic regression, controlled for clustering within hospitals and adjusted for differential sampling at one hospital, the odds of a positive slide decreased by $10 \%$ (odds ratio $0.90,95 \%$ confidence interval 0.86 to $0.94, \mathrm{P}<0.001$ ) with each 100 metre increase in altitude. Age had a significant effect in the model $(\mathrm{P}<0.001)$. Compared with children under the age of 2 years, the odds of a positive slide was higher among $2-4$ year olds (1.35, 0.96 to 1.89$)$ and then declined with age to 0.74 (0.39 to

Table 1 Patients admitted to hospital with diagnosis of malaria with at least one study criterion of severe disease by research blood slide result, age, and altitude (metres) of residence

\begin{tabular}{lccc} 
Altitude & Total $^{*}$ & No (\%) slide positive & No (\%) slide negative \\
\hline Age $<5$ years & & & \\
\hline$<600 \mathrm{~m}$ & 1392 & $958(68.8)$ & $434(31.2)$ \\
\hline $600-<1200 \mathrm{~m}$ & 1185 & $584(49.3)$ & $601(50.7)$ \\
\hline$\geq 1200 \mathrm{~m}$ & 212 & $46(21.7)$ & $166(78.3)$ \\
\hline Age $\geq \mathbf{5}$ years & & $88(19.3)$ & $369(80.7)$ \\
\hline$<600 \mathrm{~m}$ & 457 & $264(35.2)$ & $486(64.8)$ \\
\hline $600-<1200 \mathrm{~m}$ & 750 & $74(22.0)$ & $262(78.0)$ \\
\hline$\geq 1200 \mathrm{~m}$ & 336 &
\end{tabular}

*Altitude of residence missing in 141 cases, age missing in one case.

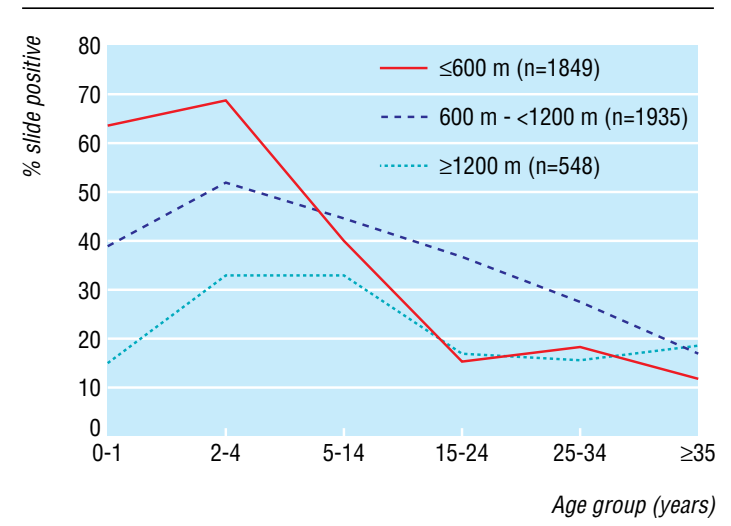

Fig 2 Percentage of patients with at least one study criterion of severe disease who had a positive research blood slide for any $P$ falciparum asexual parasites by age and altitude of residence 
Table 2 Prevalence of selected clinical features by research blood slide result and age

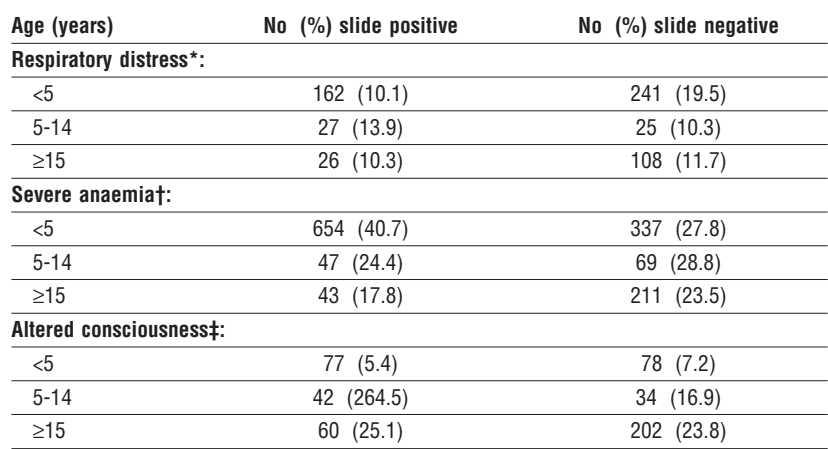

*Presence of chest indrawing (intercostals or subcostal recession) or abnormally deep breathing. Data missing or incomplete in nine cases.

† Haemoglobin $<50 \mathrm{~g} / \mathrm{l}$ from fingerprick sample. Data missing or incomplete in 80 cases. fInability to localise painful stimulus (or, in those under 1 year, no motor response to pain) in absence of hypoglycaemia (blood glucose $<2.2 \mathrm{mmol} / \mathrm{l}$ ) and without convulsion in preceding 60 minutes or administration of anticonvulsant in previous six hours. Data missing or incomplete in one case.

$1.40)$ at $5-15$ years and $0.24(0.10$ to 0.59$)$ at over 15 years. Adjustment for the reported use of antimalarial drugs in the 48 hours before admission and rainy season did not alter the effect of age and altitude and neither factor had a significant association with slide result. There was no significant difference in distribution of the three main categories of severe disease (severe anaemia, respiratory distress, and altered consciousness) between slide positive and slide negative patients stratified by age group, except that in children under the age of 5 years severe anaemia was more common among slide positive patients $(\mathrm{P}<0.001)$ and respiratory distress was more common among slide negative patients $(\mathrm{P}=0.027)$ (table 2$)$.

The unadjusted odds of dying among slide negative patients was higher than among those who were slide positive (1.85, 1.37 to $2.49, \mathrm{P}<0.001$ ), an effect observed across all age groups (table 3 ). Table 4 shows the odds ratios from a logistic regression model for mortality after adjustment for clustering within hospitals. Respiratory distress, severe anaemia, altered consciousness, age, and altitude of residence were all significantly associated with case fatality. After we controlled for these variables, those who were slide negative still had increased odds of dying but the difference was of borderline significance $(1.55,0.94$ to 2.53 , $\mathrm{P}=0.08$ ). After adjustment for the effect of the slide result, respiratory distress and altered consciousness were associated with the largest increase in the odds of a fatal outcome. Mortality increased with age and decreased with increasing altitude.

In $683 / 4474(15 \%)$ patients there were discrepancies in the reading of the research slides between the first and second research microscopists. These slides were read again by a third microscopist. When we used the definitive agreed result as the reference, only 2949/4451 hospital slides were correct $(66 \%$ agreement, $\kappa=0.33, \mathrm{P}<0.0001)$, with 988 false positive $(39 \%$ of positives) and 514 false negative (27\% of negatives). This equates to a sensitivity, specificity, and positive predictive value of hospital slides in this group of $75 \%, 59 \%$, and $61 \%$, respectively.

Of 2375 patients who were slide negative by research results, $1571(66.1 \%)$ were not treated with antibiotics in addition to the antimalarial drug. The research slide was not immediately available to clinicians as it was read later but the hospital slide result was available to clinical staff at the time of diagnosis. Patients with negative hospital slides were more likely to have received antibiotics $(661 / 1897,34.8 \%)$ compared with those with positives slides $(500 / 2499,20.0 \%)\left(\chi^{2}=122.1,2 \mathrm{df}, \mathrm{P}<0.001\right)$. Among patients with negative hospital slides, those who died were more likely to have received antibiotics than those who survived $\left(\chi^{2}=13.5,2 \mathrm{df}, \mathrm{P}<0.001\right)$ (table 5).

\section{Discussion}

\section{Patients treated for severe malaria often have no malaria parasites}

Considerable advances have been made in the management of people with febrile illness in Africa and in ensuring that those who are severely ill get to hospital. ${ }^{11-13}$ What happens when they reach hospital has received less attention, despite considerable scope for improvement. ${ }^{4}$ Malaria is the commonest reason given for admission to hospital and for death in many African countries. Even small improvements in the management of severe febrile illness classified as malaria therefore have the potential to lead to important health gains.

With the exception of children aged under 5 living in areas of high malaria transmission, most children and adults treated for malaria had no evidence of parasites on carefully examined research slides, and the chances of an episode treated as malaria actually being malaria decreased systematically with decreasing intensity of transmission. While overdiagnosis of malaria is widely suspected, it has not previously been shown systematically at different levels of malaria transmission and at different ages.

Our findings have importance for individual case management and for public health. Attempts to improve management of people diagnosed with severe malaria, however well implemented, will have little impact if most of those treated do not, in fact, have the disease. The proportion of admitted patients treated for malaria who were slide positive was similar to the local prevalence of malaria parasitaemia, ${ }^{14}$ suggesting that malaria was often used as a default diagnosis for severe febrile illness.

\section{Patients without parasites are often not treated with antibiotics}

Mortality was higher in slide negative patients than slide positive patients in all age groups, a result also found in another recent study, ${ }^{15}$ although the reduced effect in the adjusted model raises the possibility of residual confounding. Our study was not designed to determine the cause of febrile illness not caused by malaria, and we cannot determine whether the mortality in slide negative patients was preventable by antibiotic treatment, but it does identify the wider use of antibiotics as one possible

Table 3 Case fatality by research blood slide result and age among cases with at least one study criterion of severe disease

\begin{tabular}{|c|c|c|c|c|c|}
\hline \multirow[b]{2}{*}{ Age $^{\star}$ (years) } & \multirow[b]{2}{*}{ Total } & \multicolumn{2}{|c|}{ Slide positive } & \multicolumn{2}{|c|}{ Slide negative } \\
\hline & & No of cases & No $(\%)$ of deaths & No of cases & No $(\%)$ of deaths \\
\hline$<2$ & 1855 & 1016 & $67(6.6)$ & 839 & $82(9.8)$ \\
\hline $2-4$ & 996 & 598 & $27(4.5)$ & 398 & $28(7.0)$ \\
\hline $5-14$ & 441 & 196 & $21(10.7)$ & 245 & 29 (11.8) \\
\hline$\geq 15$ & 1181 & 252 & $27(10.7)$ & 929 & 153 (16.5) \\
\hline
\end{tabular}

*Age missing in one (non-fatal) case. 


\begin{tabular}{|c|c|c|c|c|}
\hline & Actual cases (deaths, \%) & Projected cases† (deaths, \%) & Odds ratio $(95 \% \mathrm{CI})$ & $P$ valueł \\
\hline \multicolumn{5}{|c|}{ Age group (years): } \\
\hline $0-1$ & $1855(149,8.0)$ & $2465(223,9.1)$ & 1.0 & \multirow[t]{4}{*}{0.057} \\
\hline $2-4$ & $996(55,5.5)$ & $1164(74,6.4)$ & 0.87 (0.56 to 1.32$)$ & \\
\hline $5-15$ & $441(50,11.3)$ & $483(52,10.8)$ & 1.15 (0.63 to 2.11$)$ & \\
\hline$\geq 15$ & $1181(180,15.2)$ & $1181(180,15.2)$ & 1.70 (1.19 to 2.42$)$ & \\
\hline \multicolumn{5}{|c|}{ Respiratory distress§: } \\
\hline No & $3876(251,6.5)$ & $4542(297,6.5)$ & 1.0 & \multirow[t]{2}{*}{$<0.001$} \\
\hline Yes & $589(179,30.4)$ & $741(228,30.8)$ & 6.61 (5.25 to 8.33$)$ & \\
\hline \multicolumn{5}{|c|}{ Severe anaemiaß: } \\
\hline No & $3033(285,9.4)$ & $3445(330,9.6)$ & 1.0 & \multirow[t]{2}{*}{0.02} \\
\hline Yes & $1361(138,10.1)$ & $1766(188,10.7)$ & 1.42 (1.06 to 1.90$)$ & \\
\hline \multicolumn{5}{|c|}{ Altered consciousness§: } \\
\hline No & $3476(229,6.6)$ & $4209(299,7.1)$ & 1.0 & \multirow[t]{2}{*}{$<0.001$} \\
\hline Yes & $493(112,22.7)$ & $517(125,24.2)$ & 3.83 (2.43 to 6.03 ) & \\
\hline \multicolumn{5}{|l|}{ Slide positiveी: } \\
\hline No & $2412(292,12.1)$ & $2661(336,12.6)$ & 1.0 & \multirow[t]{2}{*}{0.08} \\
\hline Yes & $2062(142,6.9)$ & $2633(193,7.3)$ & 1.55 (0.94 to 2.53 ) & \\
\hline \multicolumn{5}{|c|}{ Altitude of residence $(100 \mathrm{~m})^{* *}$ : } \\
\hline$<600$ & $1849(193,10.4)$ & $2616(285,10.9)$ & 0.91 (0.84 to 0.97$)$ & 0.01 \\
\hline $600-<1200$ & $1936(149,7.7)$ & $1974(152,7.7)$ & & \\
\hline$\geq 1200$ & $548(65,11.9)$ & $549(65,11.8)$ & & \\
\hline
\end{tabular}

*Based on predicted numbers of cases when adjusted for sampling methods as described in methods. Factors tested in model but found to contribute no significant effect ( $P>0.1)$ were rainy season, reported treatment for malaria before admission, literacy of patient or mother if patient $<15$ years of age.

†Predicted cases (\%) after adjustment for sampling of $50 \%$ of children under the age of 13 years admitted to one low altitude hospital; the projected numbers correspond to number of cases if sampling at this site was $100 \%$.

$\ddagger$ Wald test for linear trend.

§Case definitions as in table 2; incomplete data in 9 cases of respiratory distress, 80 cases of anaemia, and 505 cases of altered consciousness. Missing age in one case, and missing altitude of residence in 141 cases.

IResearch blood films double read for presence of any $P$ falciparum asexual parasites as described in methods.

**Model estimates for every $100 \mathrm{~m}$ increase in altitude of village of residence. Descriptive data shown for grouped altitude bands.

intervention. Two thirds (66\%) of admitted patients treated for severe malaria but with a negative hospital slide result were not treated with antibiotics, suggesting that clinicians ignored slide results. The poor correlation of hospital slides with double or triple read research slides suggests this may be justified. If clinicians are unsure of the diagnosis in a patient with severe febrile illness, it is reasonable to treat for malaria. What is more surprising is that when clinicians had doubts about the diagnosis they chose not to treat patients with antibiotics. The case fatality of those treated with antibiotics in hospital (in addition to antimalarial drugs) was higher than in those treated with antimalarial drugs alone, probably because clinicians identify the most critically ill patients for dual therapy. However, almost half (43\%) of patients who died and had a negative hospital slide result (available to clinicians at diagnosis) did not receive an antibiotic while in hospital.

It is possible, but unlikely, that our study hospitals were atypical of east African hospitals generally. The area of Tanzania where the study was conducted is stable and well ordered, and study hospitals included most district hospitals in the region. Cli- nicians knew hospital practice was being observed (possible Hawthorn effect) and any alteration to their practice as a result of the study was likely, if anything, to have stimulated more careful diagnosis. The clear cut nature of the measures (age, altitude of residence, pattern of disease, outcome, and double read blood slides) and the consistency of the findings both internally and with other studies from east Africa also suggest the findings are robust. ${ }^{16}{ }^{17}$

True slide negative cases of severe malaria are rare (although it can be confirmed only by postmortem examination). A positive slide is, however, a common incidental finding in areas where many people are semi-immune to malaria and have asymptomatic parasite carriage. The predictive value of positive slides for clinical malaria may be improved by considering parasite density. ${ }^{18}$ If our definition of malaria had included a parasite density of $>1000$ asexual $P$ falciparum parasites per $\mu$, the number of cases defined as not malaria would have increased by about $15 \%$. We therefore think our estimate of overdiagnosis of malaria is conservative.

Table 5 Number (\%) of all cases and fatal cases treated with any antibiotic during hospital admission, according to hospital blood slide result and research blood slide result

\begin{tabular}{|c|c|c|c|c|}
\hline & \multicolumn{2}{|c|}{ All cases* } & \multicolumn{2}{|c|}{ Fatal cases } \\
\hline & No of patients & Not treated with antibiotic & No of patients & Not treated with antibiotic \\
\hline \multicolumn{5}{|c|}{ Hospital slide resultt: } \\
\hline Negative & 1897 & $1236(65.2)$ & 248 & $99(39.9)$ \\
\hline Positive & 2499 & $1999(80.0)$ & 174 & $101(58.0)$ \\
\hline \multicolumn{5}{|c|}{ Research slide resultł: } \\
\hline Negative & 2375 & $1571(66.1)$ & 290 & $120(41.4)$ \\
\hline Positive & 2043 & $1680(82.2)$ & 142 & $86(60.6)$ \\
\hline
\end{tabular}

${ }^{*}$ Antibiotic treatment refers to record of antibiotic treatment during hospital admission; all cases were treated with antimalarial.

†Data on antibiotic treatment of hospital slide result missing in 78 cases (including 12 fatal cases).

†Data on antibiotic treatment missing in 56 cases (including two fatal cases). 


\section{What is already known on this topic}

Falciparum malaria is a major cause of morbidity and mortality in Africa

Around half of all hospital admissions in much of Africa are attributed to malaria

Diagnostic facilities in most hospitals in Africa are limited, and diagnosis of many diseases, including malaria, is often inaccurate

Certain presentations, especially raised respiratory rate and reduced level of consciousness, are predictive of poor outcome in children with malaria

\section{What this study adds}

Except for children in areas of high transmission, children diagnosed and treated for severe malaria often have no parasites on their blood film

Most adults at every transmission level who are treated for severe malaria do not have laboratory evidence of disease

Many of those with severe febrile illness with no malaria parasites are not treated with antibiotics

The syndromes associated with poor outcome in severe childhood malaria are also predictive of poor outcome in febrile children without malaria, and in adults with and without malaria

As in previous studies, respiratory distress and altered consciousness were the strongest predictors of mortality ${ }^{16}{ }^{17}$ Our findings emphasise that these associations with mortality apply to both severe malaria and other severe febrile illness and to adults as well as children.

\section{Conclusion}

Overdiagnosis of malaria is widespread and an important part of the need to improve basic standards of hospital care in Africa, where massive need has to be met with limited resources. Current evidence suggests that many of the existing guidelines are not followed, ${ }^{4}$ laboratory workload compromises quality, ${ }^{19}$ and triaging hospital admissions to prioritise care is rarely practised. ${ }^{5}$ One option is to try to improve diagnostic accuracy, although this is not easy. An alternative is to move to a more syndromic approach to hospital care in Africa, concentrating efforts on treating the probable causes of severe febrile illness. ${ }^{20}{ }^{21}$ For severe febrile disease the most effective approach is likely to depend on the setting and on the true incidence of malaria. We have shown that this is a major priority in all age groups and at all transmission intensities. Efforts to improve the management of clinically diagnosed severe malaria are as important in areas of low transmission and in adults as in the young children in high transmission areas who constitute the majority of the over one million deaths from malaria every year.

We thank the patients and their families who participated in this study. We also thank the clinical and laboratory staff who assisted in data collection: Mark Swai, Werner Shimana, Ole Nguyaine, Cleopa Mbwambo, Justina Mushi, Francis Assenga, Joseph Minja, Alan Minja, William Silayo, Richard Mcharo, Raymond Urassa, Richard Collins, Hilda Mbakilwa, Sia Nelson, Nsia Muro, Elizabeth Msoka, Theresia Mtui, Sarah Mushi, Michael Irira, Esther Lyatu, Alutu Masokoto, Frank Magogo, Nico Funga, Lincoln Male, William Chambo, and Zacharia Zafaelli.
Contributors: HR, RM, CD, and CJMW were involved in design, implementation, analysis, and writing up. IC and OM were responsible for data analysis, writing up of the results, and making critical revisions to the manuscript. $\mathrm{KS}$ and EM were each responsible for clinical data collection in the largest sites and made critical revisions on the paper. JS, AK, BMG, and RO were actively involved in the design of the study and planning the analysis and made critical revisions to the manuscript. Eleanor Riley, Daniel Chandramohan, Jon Cox, W M M Nkya, Martha Lemnge, Thor Theander, Joanna Schellenberg, and Jane Bruce also contributed to design, analysis, and reviewing the manuscript. HR is guarantor.

Funding: Medical Research Council, UK (grant No 9901439). CJMW is supported by the Gates Malaria Partnership.

Competing interests: None declared.

Ethical approval: The ethical committees of the National Institute of Medical Research, Tanzania, and the London School of Hygiene and Tropical Medicine approved the study.

1 Ministry of Health. Health statistics abstract 2002. Burden of disease and health utilization statistics. Vol 1. Dar Es Salaam, Tanzania: Ministry of Health, 2002:3-3.

World Health Organization. The Africa malaria report. Geneva: WHO, 2003:1093. (WHO/CDS/MAL/2003.)

3 Makani J, Matuja W, Liyombo E, Snow RW, Marsh K, Warrell DA. Admission diagnosis of cerebral malaria in adults in an endemic area of Tanzania: implications and clinical description. OJM 2003;96:355-62.

4 English M, Esamai F, Wasunna A, Were F, Ogutu B, Wamae A, et al. Assessment of inpatient paediatric care in first referral level hospitals in 13 districts in Kenya. Lancet 2004;363:1948-53.

5 Nolan T, Angos P, Cunha AJ, Muhe L, Qazi S, Simoes EA, et al. Quality of hospital care for seriously ill children in less-developed countries. Lancet 2001;357:106-10.

6 Jonkman A, Chibwe RA, Khoromana CO, Liabunya UL, Chapanda ME, Kandiero GE, et al. Cost-saving through microscopy-based versus presumptive diagnosis of malaria in adult outpatients in Malawi. Bull World Health Organ 1995;73:223-7.

7 Snow RW, Craig M, Deichmann U, Marsh K. Estimating mortality, morbidity and disability due to malaria among Africa's non-pregnant population. Bull World Health Orsability due to malari

8 Bodker R, Akida J, Shayo D, Kisinza W, Msangeni HA, Pedersen EM, et al. Relationship between altitude and intensity of malaria transmission in the Usambara Mountains, Tanzania. J Med Entomol 2003;40:706-17.

9 WHO. Severe falciparum malaria. Trans R Soc Trop Med Hyg 2000;94:1-2

10 Molyneux ME, Taylor TE, Wirima JJ, Borgstein A. Clinical features and prognostic indicators in paediatric cerebral malaria: a study of 131 comatose Malawian children. $Q J$ Med 1989;71:441-59.

11 WHO. Inegrated management of childhood illness. www.who.int/child-adolescenthealth/publications/IMCI/WHO_FCH_CAH_00.40.htm (accessed 7 Oct 2004).

12 Bryce J, el Arifeen S, Pariyo G, Lanata C, Gwatkin D, Habicht JP. Reducing child mortality: can public health deliver? Lancet 2003;362:159-64.

13 World Health Organization. Africa malaria report 2003. Geneva: WHO, 2003. World Health Organization. Africa
(WHO/CDS/MAL/2003.1093 ed.)

14 Bodker R. Variations in malaria risk in the Usambara Mountains, Tanzania. Bodker R. Variations in malaria risk in the Usambara Mountai
Charlottenlund, Denmark: Danish Bilharzia Laboratory, 2000:56-84.

15 Planche T, Agbenyega T, Bedu-Addo G, Ansong D, Owusu-Ofori A, Micah F, et al. A prospective comparison of malaria with other severe diseases in African children: prognosis and optimization of management. Clin Infect Dis 2003;37:890-7.

16 Marsh K, Forster D, Waruiru C, Mwangi I, Winstanley M, Marsh V, et al. Indicators of life-threatening malaria in African children. $N$ Engl J Med 1995;332:1399-404.

17 Schellenberg D, Menendez C, Kahigwa E, Font F, Galindo C, Acosta C, et al. African children with malaria in an area of intense Plasmodium falciparum transmission: features on admission to the hospital and risk factors for death. Am J Trop Med Hyg tures on admission

18 Smith T, Schellenberg JA, Hayes R. Attributable fraction estimates and case definitions for malaria in endemic areas. Stat Med 1994;13:2345-58

19 Mundy C, Ngwira M, Kadewele G, Bates I, Squire SB, Gilks CF. Evaluation of microscope condition in Malawi. Trans R Soc Trop Med Hyg 2000;94:583-4.

20 WHO. Management of the child with severe infection or severe malnutrition. Geneva: WHO, 2000

21 English M, Berkley J, Mwangi I, Mohammed S, Ahmed M, Osire F, et al. Hypothetical performance of syndrome-based management of acute paediatric admissions of children aged more than 60 days in a Kenyan district hospital. Bull World Health Organ 2003;81:166-73

(Accepted 8 September 2004)

doi $10.1136 /$ bmj.38251.658229.55

London School of Hygiene and Tropical Medicine, London WCIE 7HT

Hugh Reyburn clinical senior lecturer

Chris Drakeley parasitologist

Ilona Carneiro statistician

Brian M Greenwood professor

Christopher J M Whitty clinical senior lecturer

Kilimanjaro Christian Medical Centre, Moshi, Tanzania

Redepmta Mbatia clinical epidemiologist

Ombeni Mwerinde data manager

John Shao executive director

Raimos Olomi professor

National Institute of Medical Research, Dar es Salaam, Tanzania

Emmanuel Mwakasungula clinician 
Andrew Kitua director general

Mawenzi Hospital, Moshi, Kilimanjaro, Tanzania
Kapalala Saganda hospital superintendent

Correspondence to: H Reyburn hugh.reyburn@lshtm.ac.uk

N 